\title{
Correlation of glycated albumin with self blood glucose monitoring in diabetic patients on hemodialysis taking erythropoietin
}

\author{
Khalidah M Bador ${ }^{\mathrm{a}}$, Sharifah KA Kamaruddin ${ }^{\mathrm{b}}$, Norita T Yazid ${ }^{\mathrm{b}}$ \\ ${ }^{a}$ Department of Pathology, Faculty of Medicine, Universiti Kebangsaan Malaysia Medical Centre, \\ Cheras 56000, Kuala Lumpur, ${ }^{b}$ Department of Pathology, Selayang Hospital, 68100 Batu Caves, \\ Selangor, Malaysia
}

\begin{abstract}
Background: Serum glycated albumin (GA) is a marker of glycemic control in diabetic renal patients, but studies were limited by the use of few random glucose values to define glycemic control.

Objectives: To determine whether GA correlated with self blood glucose monitoring is better than HbA1c in hemodialyzed diabetic patients taking erythropoietin.

Methods: This was a cross-sectional study of diabetic patients on hemodialysis with and without erythropoietin. GA was measured by ELISA and HbA1c by ion-exchange HPLC. GA was reported as the GA/albumin ratio where albumin was measured using bromocresol green. The average capillary blood glucose level over the preceding three months (CBG) was calculated from self-reported daily prebreakfast, prelunch, and prebed glucose meter readings.

Results: Thirty-four patients were recruited; 18 were taking erythropoietin (6000 units per week) and 16 had never received erythropoietin. HbA1c correlated poorly with CBG in patients taking erythropoietin $(r=-0.014$, $P=0.96)$ compared with patients without erythropoietin $(r=0.579, P=0.02)$. The correlation of GA/albumin ratio with CBG in the erythropoietin group $(r=0.612, P=0.007)$ was similar to the nonerythropoietin group $(r=0.854, P<0.001)$. The slope for HbA1c versus CBG was 2.8-fold greater in patients without erythropoietin compared with those taking erythropoietin. There was no significant difference in the slopes for GA/albumin ratio versus CBG between the two patient groups $(P>0.05)$.

Conclusion: In diabetic patients on hemodialysis and taking low doses of erythropoietin, GA/albumin is a better marker of glycemic control than HbA1c.
\end{abstract}

Keywords: Erythropoietin, glycated albumin, glucose monitoring, hemodialysis

Recent studies have shown that HbA1c is an inadequate marker of hyperglycemia in diabetic patients with chronic kidney disease (CKD). HbA1c levels were lower in diabetic patients on peritoneal or hemodialysis compared with their counterparts without kidney impairment [1-4]. These same studies provided evidence that glycated albumin (GA) correlated better with glucose levels in this group of diabetic patients.

Chronic renal failure is associated with anemia, altered protein metabolism and proteinuria. GA was not affected by factors that affect hemoglobin turnover

Correspondence to: Khalidah M Bador, Department of Pathology, Faculty of Medicine, Universiti Kebangsaan Malaysia Medical Centre, Cheras 56000, Kuala Lumpur, Malaysia.E-mail: khalidah@ppukm.ukm.edu.my such as anaemia and erythropoietin treatment [2, 3]. Diabetic subjects with proteinuria did not have significantly lower GA levels compared to subjects with microalbuminuria [5]. Globally, the dosage of erythropoietin used in CKD patients varies widely. Weekly doses in American dialysis patients are a few hundred-fold higher than weekly doses given to Asian patients. The use of erythropoietin has been associated with lower HbA1c levels in a dose-dependent manner [2]. Nevertheless, in a study of Japanese diabetic patients on hemodialysis, HbA1c underestimated glycemic control even at low doses of erythropoietin [6].

An important limitation of previous studies concerns the glucose measurements used to define glycemic control [7]. In most studies, glycemic control was based on one, two, or three values of random 
plasma glucose levels taken during the months before GA measurement. One study used a single-day sevensample glucose profile set [1], which was the reference method used in the Diabetes Control and Complications Trial. In another study the authors compared HbA1c with plasma glucose levels during a $75 \mathrm{~g}$ oral glucose tolerance test [8]. However, because of their metabolic turnover rates, HbA1c and GA reflect glycemic status over two to three months and four to six weeks respectively.

Self blood glucose monitoring (SBGM) has been recommended as another way to monitor glycemic control. Therefore, the aim of this study was to determine whether GA or HbA1c correlated better with SBGM in hemodialyzed diabetic patients taking erythropoietin.

\section{Materials and methods}

This was a cross-sectional study of diabetic patients undergoing hemodialysis with and without erythropoietin therapy. All diabetic subjects were recruited at the hospital's dialysis unit after obtaining their informed written consent. The study received approval from our institutional ethics committees. Patients who had received blood transfusion or had changes to their antidiabetic medications within the previous three months were excluded, as well as patients with liver disease, malignancy, or thyroid disorders.

Self-reported daily prebreakfast, prelunch, and prebed glucose meter readings were extracted from the patients' record book. The average capillary blood glucose level over the three months prior to recruitment (CBG) was calculated. Demographic and clinical data (age, gender, ethnicity, and erythropoietin dose) were obtained from the medical records. Venous whole blood samples for HbA1c, GA, and albumin assays were taken prior to hemodialysis. HbA1c and albumin were measured within one day of sample collection. Serum samples for GA were stored at $-70^{\circ} \mathrm{C}$ prior to analysis.

HbA1c was measured by an automated ionexchange HPLC method (BioRad D10, USA). Samples with abnormal chromatograms that suggested the presence of hemoglobinopathies or other interferences were excluded. HbA1c was reported as the percentage of total hemoglobin with a nondiabetic reference range of $4.0 \%-6.3 \%$. GA was measured by ELISA (Cusabio Biotech, Germany) with a detection range of 78-5000 nmol/l. Albumin was measured using a bromocresol green method on an automated instrument (AU2700, Beckman Counter, USA). The GA to albumin ratio (GA/albumin ratio) was calculated to correct for albumin differences between subjects.

\section{Statistical analyses}

Descriptive data of patient groups were reported as numbers of patients (categorical data) or median and interquartile range (continuous data). KruskalWallis and Mann-Whitney $U$ tests were used to identify differences between patient groups. Correlations between CBG and HbA1c and GA/ albumin ratio were determined by Spearman's correlation coefficient. The difference in the slopes between patient groups was tested by ANOVA. All statistical analyses were performed using the Statistical Package for Social Sciences (USA) and differences with $P<0.05$ were considered as significant.

\section{Results}

Thirty-four diabetic patients on hemodialysis were studied; 18 patients received a weekly dose of 6000 units of erythropoietin, while 16 had never received erythropoietin (Table 1). There was no significant difference in age, sex, or ethnicity between the patient groups. Patients taking erythropoietin had a higher CBG and GA/albumin ratio, but lower HbA1c than patients without erythropoietin. However the difference between the patient groups was only significant for the GA/albumin ratio $(P=0.036)$.

The correlations between HbA1c and GA/albumin ratio with CBG in the two patient groups were examined (Figure 1). In patients taking erythropoietin, there was a weak correlation between HbA1c and CBG $(r=-0.014, P=0.956)$ compared with patients without erythropoietin $(r=0.579, P=0.019)$. The correlation of GA/albumin ratio with CBG in the erythropoietin group ( $r=0.612, P=0.007)$ was comparable with the nonerythropoietin group ( $r=0.854, P<0.001$ ), and the correlations were stronger compared with HbA1c.

The slope (rate of increase) for HbA1c versus CBG was 2.8-fold higher in patients without erythropoietin compared with those treated with erythropoietin. There was no significant difference in the slopes for the GA/albumin ratio versus CBG between the two patient groups $(P>0.05)$. 
Table 1. Group characteristics in diabetic patients undergoing hemodialysis

\begin{tabular}{llll}
\hline & $\begin{array}{c}\text { Non EPO } \\
(\mathbf{n}=\mathbf{1 6})\end{array}$ & $\begin{array}{c}\text { With EPO } \\
(\mathbf{n}=\mathbf{1 8})\end{array}$ & 0.14 \\
\hline Sex & & & \\
$\quad$ Male & 12 & 9 & 0.21 \\
$\quad$ Female & 4 & 9 & \\
Race & & & \\
$\quad$ Malay & 9 & 7 & 0.13 \\
$\quad$ Chinese & 6 & 4 & 0.48 \\
$\quad$ Indian & 1 & $58.0(9)$ & 0.10 \\
Age, y & $61.5(21)$ & $13.5(6.7)$ & 0.16 \\
CBG, mmol/L & $11.8(4.5)$ & $7.1(2.9)$ & 0.06 \\
HbA1c, \% & $9.0(3.4)$ & $2555(1058)$ & 0.036 \\
GA, nmol/L & $2152(747)$ & $40(7)$ & $69.7(25.1)$ \\
Albumin, g/L & $44(6)$ & & \\
GA/albumin ratio & $50.0(21.8)$ & & \\
& & & \\
\hline
\end{tabular}

Values are numbers of patients or median values (interquartile range). $\mathrm{EPO}=$ erythropoietin, $\mathrm{CBG}=$ three months averaged capillary blood glucose, GA = glycated albumin
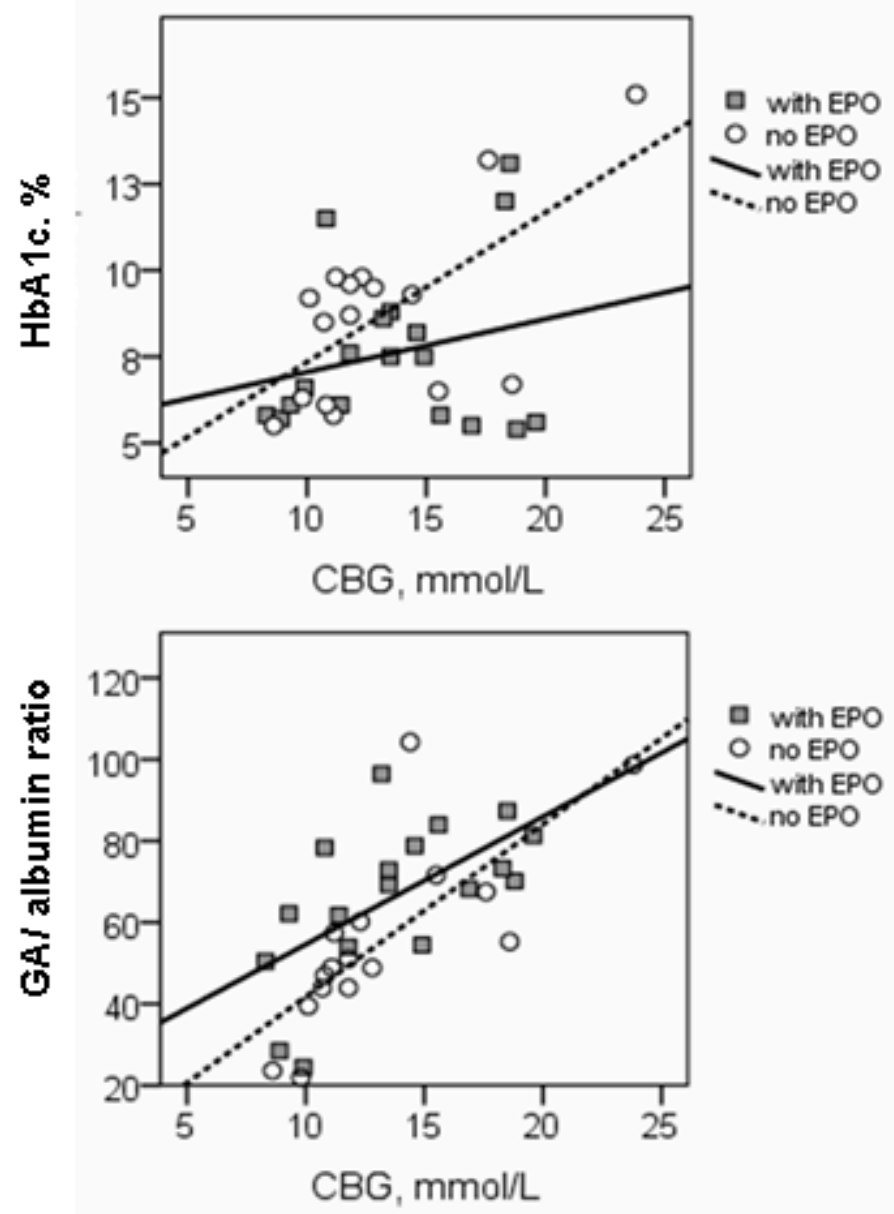

Figure 1. Correlations of HbA1c (upper panel) and GA/albumin ratio (lower panel) with CBG in diabetic patients on hemodialysis 


\section{Discussion}

HbA1c was established as a biomarker of hyperglycemia by large clinical trial studies that demonstrated that tight glucose control in diabetes delays progression of microvascular complications such as nephropathy $[9,10]$. The limitation of using HbA1c in patients with anemia and hemoglobinopathies is well documented [11]. False results have also been reported with uremia as a result of interference by carbamylated hemoglobin although this effect is method-dependent.

In diabetic patients with advanced kidney disease an additional factor is the use of erythropoietin. Erythropoietin increases red cell turnover and results in a higher proportion of reticulocytes in the blood circulation. Consequently, HbA1c tends to underestimate glycemic status [12]. Erythropoietin has no effect on albumin metabolism and thus measurement of GA might be expected to overcome the problems associated with erythropoietin use.

In this study, patients taking erythropoietin had higher median levels of capillary blood glucose and GA/albumin ratio with lower HbA1c. However, only the GA/albumin ratio was statistically different between the patient groups. This may be the result of the low dose of erythropoietin in this population and the small number of subjects. Nevertheless, HbA1c correlated poorly with CBG in patients taking erythropoietin, whereas the correlations of GA/albumin ratio with CBG were comparable among patients with and without erythropoietin. Based on the divergence in slopes, HbA1c can start to underestimate hyperglycemia when glucose levels are above 10 $\mathrm{mmol} / \mathrm{L}$ in patients given low dose erythropoietin. This finding is similar to the results from the study in Japanese diabetics where the average dose of erythropoietin was $4000 \mathrm{U}$ per week [6]. Patients in the highest quartile of \%GA with an average random glucose of $8.8 \mathrm{~mol} / \mathrm{l}$ had two times higher \%GA compared with those in the first quartile (average glucose $6.7 \mathrm{mmol} / \mathrm{l}$ ) with no difference in HbA1c. However, the Japanese study compared GA to a single nonfasting glucose sample.

One of the aims of this study was to obtain sufficient glucose values that would reflect long-term variation of glycemic control. SBGM has been recommended in several clinical practice guidelines. This approach relies on self-reported glucose levels measured using glucose meters. In general, results from glucose meters are not as accurate as laboratory methods although, under optimal conditions, many meters have been shown to meet the analytical accuracy recommended [12]. Secondly, whole blood has slightly lower glucose values compared with plasma. Glucose meters are therefore calibrated to report results equivalent to plasma, but assume a normal hematocrit.

Continuous blood glucose monitoring is an alternative, but this invasive method and not well standardized [14]. In a recent study, GA correlated significantly with mean interstitial glucose concentration as measured by continuous glucose monitoring over 48 hours. Because of the short duration, only subjects with stable glycemic control for six months prior to GA measurement were studied and the continuous monitoring method was verified against a 7-point glucose profile [15]. By comparison, our study evaluated GA against SBGM to reflect the fluctuations of glucose levels and long exposure time of albumin to hyperglycemia in the circulation.

Comparison of HbA1c and GA is inherently difficult because they reflect hyperglycemia over different time intervals. HbA1c is generally considered to reflect mean plasma glucose levels over the previous two to three months. However, changes in glycemic control during the last one month contribute a greater proportion of the HbA1c result [16]. GA reflects glucose levels over a few weeks. In theory, HbA1c values derived from hemoglobins with a higher turnover rate become comparable to GA. This prediction is confounded by altered metabolism of both hemoglobin and albumin in chronic renal failure. In this study, a three month period to assess glycemic control was selected in order to compare with current clinical practice of quarterly measurements of glycosylated hemoglobin.

Recent reports have suggested that HbA1c and GA correlate more strongly with postprandial glucose than mean plasma glucose $[17,18]$. The glucose value used in this study was the average of one fasting (prebreakfast) and two random (prelunch and prebed) glucose readings. We did not evaluate the correlations with fasting and random glucose separately because of the small number of data.

In this study we measured GA using an immunoassay with an antibody specific for human glycated albumin. The molecular form of the glycosylated albumin is highly variable [19] and therefore results were reported in nonmass units. Previous studies usually measured GA using an 
enzymatic assay (Asahi Kasei) with the results reported in mass units. Because of this methodological difference, we cannot compare absolute values of GA/ albumin ratio with \%GA values from previous reports.

A strength of this study is that we used a large number of glucose readings per individual over a 3-month period to establish glycemic control. We also reported the GA to albumin ratio, therefore compensating for any factors that may influence albumin concentration. However, the study was conducted in a small number of patients from a single institution and needs to be extended to a wider population. Further studies are also required to establish GA/albumin ratio cut-off values to stratify good and poor glycemic control as has been done for HbA1c.

\section{Conclusion}

Serial blood glucose measurements obtained with self blood glucose monitoring allow the effect of biological fluctuations on GA and HbA1c to be evaluated. In diabetic patients on hemodialysis and taking low doses of erythropoietin, GA/albumin showed a better correlation with the 3-month average of SBGM compared with HbA1c. The use of a GA/ albumin ratio to monitor this group of patients should be considered.

\section{Acknowledgments}

This study was supported by Research Grant FF0262011 from Universiti Kebangsaan Malaysia (The National University of Malaysia). The authors have no conflict of interest to report.

\section{References}

1. Chujo K, Shima K, Tada H,Oohashi T, Minakuchi J, Kawashima S. Indicators for blood glucose control in diabetics with end-stage chronic renal disease: GHb vs. glycated albumin (GA). J Med Invest. 2006; 53:223-8.

2. Inaba M, Okuno S, Kumeda Y, Yamada S, Inamishi Y, Tabata. T, et al. Glycated albumin is a better glycemic indicator than glycated hemoglobin values in hemodialysis patients with diabetes: effect of anemia and erythropoietin injection. J Am Soc Nephro. 2007; 18:895-903.

3. Peacock TP, Shihabi ZK, Bleyer, AJ Dolbare EL, Byers JR, Krovich MA, et al. Comparison of glycated albumin and hemoglobin $\mathrm{A}_{1 \mathrm{c}}$ levels in diabetic patients on hemodialysis. Kidney Int. 2008; 73:1062-8.
4. Freedman BI, Shenoy RN, Planer JA,Clay KD, Shihabi ZK, Burkart JM, et al. Comparison of glycated albumin and hemoglobin $\mathrm{A}_{1 \mathrm{c}}$ concentrations in diabetic subjects on peritoneal and hemodialysis. Perit Dial Int. 2010; 30:72-9.

5. Viswanathan V, Kumpatla S, Tilak P, Muthukumaran P. Levels of glycated albumin at different stages of diabetic nephropathy in India. Int J Diabetes Metab. 2009; 17:77-80.

6. Uzu T, Hatta T, Deji N, Izumiya T, Ueda H, Miyazawa I, et al. Target for glycemic control in type 2 diabetic patients on hemodialysis: effects of anemia and erythropoietin injection on hemoglobin $A_{1 c}$. Ther Apheresis and Dialysis. 2009; 13:89-94

7. Mehrotra R, Kalantar-Zadeh K, Adler S. Assessment of glycemic control in dialysis patients with diabetes: glycosylated hemoglobin or glycated albumin? Clin J Am Soc Nephrol. 2011; 6:1520-2.

8. Nagayama H, Inaba M, Okabe R, Emoto M, Ishimura E, Okazaki S, Nishizawa Y, et al. Glycated albumin as an improved indicator of glycemic control in hemodialysis patients with type 2 diabetes based on fasting plasma glucose and oral glucose tolerance test. Biomed Pharmacother. 2009; 63:236-40.

9. The Diabetes Control and Complications Trial (DCCT) Research Group. The effect of intensive treatment of diabetes on the development and progression of long-term complications in insulin-dependent diabetes mellitus. N Engl J Med. 1993; 329:977-86.

10. UK Prospective Diabetes Study (UKPDS) group. Intensive blood-glucose control with sulphonylureas or insulin compared with conventional treatment and risk of complications in patients with type 2 diabetes (UKPDS 33). Lancet. 1998; 352(9131):837-53.

11. Bry L, Chen PC, Sacks DB. Effects of hemoglobin variants and chemically modified derivatives on assays for glycohemoglobin. Clin Chem. 2011, 47:453-63

12. Nakao T, Matsumoto H, Okada T, Han M, Hidaka H, Yoshino M, et al. Influence of erythropoietin treatment on hemoglobin $\mathrm{A}_{1 \mathrm{c}}$ levels in patients with chronic renal failure on hemodialysis. Intern Med. 1998; 37: 826-30.

13. Johnson RN, Baker JR. Error detection and measurement in glucose monitors. Clin Chim Acta. 2001; 307:1-67

14. Riveline JP, Teynie J, Belmouaz S, Franc S, Dardari D, Bauwens M, et al. Glycaemic control of type 2 diabetic patients on chronic haemodialysis: use of a continuous glucose monitoring system. Nephrol Dial Transplant. 2009, 24:2866-71

15. Vos FE, Schollum JB, Coulter CV, Manning PJ, Duffull 
SB, Walker RJ. Assessment of markers of glycaemic control in diabetic patients with chronic kidney disease using continuous glucose monitoring. Nephrology. 2012; 17:82-188.

16. Tahara Y, Shima K. The response of GHb to step-wise plasma glucose change over time in diabetic patients. Diabetes Care. 1993; 16:1313-4

17. Yoshiuchi K, Matsuhisa M, Katakami N Nakatani Y, Sakamoto K, Matsuoka T, et al. Glycated albumin is a better indicator for glucose excursion than glycated hemoglobin in type 1 and type 2 diabetes. Endocr J.
2008; 55:503-7.

18. Suwa T, Ohta A, Matsui T, Koganei R, Kato H, Kawata $\mathrm{T}$, et al. Relationship between clinical markers of glycemia and glucose excursion evaluated by continuous glucose monitoring (CGM). Endocr J. 2010; 57:135-40.

19. Kisugi R, Kouzuma T, Yamamoto T, Akizuki S, Miyamoto H, Someya Y, et al. Structural and glycation site changes of albumin in diabetic patient with very high glycated albumin. Clin Chim Acta. 2007; 382: 59-64. 